Article

\title{
The Role of EjSPL3, EjSPL4, EjSPL5, and EjSPL9 in Regulating Flowering in Loquat (Eriobotrya japonica Lindl.)
}

\author{
Yuanyuan Jiang ${ }^{1,+} \oplus$, Jiangrong Peng ${ }^{1,+}$, Man Wang ${ }^{1}$, Wenbing Su ${ }^{1}$, Xiaoqing Gan ${ }^{1}$, Yi Jing ${ }^{2}$, \\ Xianghui Yang ${ }^{1}\left(\mathbb{D}\right.$, Shunquan Lin ${ }^{1, *}$ and Yongshun Gao ${ }^{1,3, *}$ \\ 1 State Key Laboratory for Conservation and Utilization of Subtropical Agro-bioresources, College of \\ Horticulture, South China Agricultural University, Wushan Road 483, Tianhe District, Guangzhou 510642, \\ China; yyjiang613@163.com (Y.J.); jiangrongp@163.com (J.P.);wm014527@163.com (M.W.); \\ suwenbing13@163.com (W.S.); ganxq0702@163.com (X.G.); gzyxh@scau.edu.cn (X.Y.) \\ 2 BGI Genomics, BGI-Shenzhen, Shenzhen 518083, China; shenqiandelan@live.cn \\ 3 Beijing Academy of Forestry and Pomology Sciences, Beijing Academy of Agriculture and Forestry Sciences, \\ Beijing 100093, China \\ * Correspondence: loquat@scau.edu.cn (S.L.); yongshungao@163.com (Y.G.); Tel.: +86-13380055716 (S.L.); \\ $+86-15692001878$ (Y.G.) \\ $+\quad$ These authors contributed equally to this work.
}

Received: 19 October 2019; Accepted: 16 December 2019; Published: 30 December 2019

\begin{abstract}
The age pathway is important for regulating flower bud initiation in flowering plants. The major regulators in this pathway are miR156 and SPL transcription factors. To date, SPL genes have been identified in many species of plants. Loquat, as a woody fruit tree of Rosaceae, is unique in flowering time as it blooms in winter. However, the study of its SPL homologous genes on the regulation mechanism of flowering time is still limited. In this study, four SPL homologs-EjSPL3, EjSPL4, EjSPL5, and EjSPL9—are cloned from loquat, and phylogenetic analysis showed that they share a high sequence similarity with the homologues from other plants, including a highly conserved SQUAMOSA promoter binding protein (SBP)-box domain. EjSPL3, EjSPL4, EjSPL5 are localized in the cytoplasm and nucleus, and EjSPL9 is localized only in the nucleus. EjSPL4, EjSPL5, and EjSPL9 can significantly activate the promoters of EjSOC1-1, EjLFY-1, and EjAP1-1; overexpression of EjSPL3, EjSPL4, EjSPL5, and EjSPL9 in wild-type Arabidopsis thaliana can promote flowering obviously, and downstream flowering genes expression were upregulated. Our work indicated that the EjSPL3, EjSPL4, EjSPL5, and EjSPL9 transcription factors are speculated to likely participate in flower bud differentiation and other developmental processes in loquat. These findings are helpful to analyze the flowering regulation mechanism of loquat and provide reference for the study of the flowering mechanism of other woody fruit trees.
\end{abstract}

Keywords: loquat; SPL transcription factor; flowering time; aging pathway

\section{Introduction}

Flowering is regulated by extensive factors, such as plant hormones, photoperiod, vernalization, age, temperature, light quality, sucrose, etc. [1-6]. Genes regulating flowering time have been characterized in many species, and they interact to form a complicated regulatory network [7-9]. FLOWERING LOCUS T (FT) is a crucial integrator in this network [10], and the flowering time regulating gene SUPPRESSOR OF OVEREXPRESSION OF CO1 (SOC1), the floral meristem identity genes APETALA1 (AP1), LEAFY (LFY), and FRUITFULL (FUL) are located at downstream of the regulatory network $[11,12]$. The aging pathway is reported to be a universal approach to control the transition 
from vegetative to reproductive stage, and it is primarily coordinated by microRNA156 (miR156) and its target genes SQUAMOSA PROMOTER BINDING-LIKE (SPL) transcription factors [13,14]. MicroRNAs (miRNAs) are non-coding, small-molecule RNAs of about 16-29 nt in length, are ubiquitous in organisms, and they could regulate gene expression by mediating targeted mRNA degradation or translational repression [15,16]. The aging pathway mainly involves miR156 and miR172 [14]. The targets of miR156 are the SPL transcription factors which were first discovered and confirmed in Antirrhinum majus, and were named SQUAMOSA PROMOTER BINDING PROTEIN (SBP) because they recognize and bind to the promoter region of MADS-box genes SQUAMOSA (SQUA) [17]. SBP genes were also found in green plants and referred to as the SQUAMOSA PROMOTER BINDING-LIKE $(S P L)$ genes [18]. In recent decades, the $S B P$ gene families were characterized in model plants, woods, crops, and fruits, such as Arabidopsis thaliana [19], barley [20], green algae [21], moss [22], tomato [23], salvia [24], rice [25], birch [26], light birch [27], cotton [28], grape [29], apple [30], corn [31], and so on. All SPL genes contain a highly conserved DNA binding domain, which was called the SBP-box domain, and it consists of approximately 80 amino acid residues including two zinc finger structures and one nuclear localization signal [32].

The aging pathway regulates flowering under uninduced conditions, and also, it can be integrated with other pathways [9]. In young Arabidopsis thaliana, miR156 is in a higher expression level, which negatively regulates the target SPLs; as the plant grows, the expression level of miR156 decreases, and the target SPLs mRNA increases subsequently [13]. Among the SPLs, SPL9 and SPL15 positively regulate miR172, which inhibits the expression of transcription factor APETELA2 (AP2); AP2 inhibits the expression of $F T$ and; therefore, delays flowering ultimately [33,34]. At the same time, SPL2, SPL3, SPL4, SPL5, SPL9, SPL10, SPL11, SPL13, and SPL15 can directly promote the expression of AP1, $L F Y$, and FUL, and SPL3, SPL4, and SPL5 can also recruit FT-FD complex and bind to the promoter regions of $A P 1, L F Y$ and FUL and consequently initiate their transcription [13,35]. Furthermore, SPL2, SPL9, SPL10, SPL11, SPL13, and SPL15 directly regulate SUPPRESSOR OF OVEREXPRESSION OF CONSTANS 1 (SOC1), and then activate the expression of AP1, LFY, and FUL. SOC1 also negatively regulates SPL3, SPL4, and SPL5 to form a feedback loop [13]. At present, the functions of SPL genes in more and more species has been unearthed: Grape $V p S B P 11$ can regulate floral transition and leaf development [36]; BlSPLs in Betula luminifera interact with DELLA protein to participate gibberellin regulation [27]. It is reported that, except flowering, SPL genes are vital in also other physiological processes in plants, such as adventitious root development [13,37], fertility [38], fruit growth and quality [39], floral organ and shoot development [40,41], plant hormone response [42], morphological differentiation [43], and stresses response [44].

Loquat (Eriobotrya japonica Lindl.) is a subtropical evergreen fruit that belongs to Maloideae subfamily of Rosaceae. Compared to its relative fruit trees like apple, pear, and peach, loquat has unique habits, in that it blooms in winter and fruits mature in late spring or early summer [45]. To date, some crucial flowering-related genes from loquat have been cloned. EjTFL1 and EjLFY were identified earliest, and EjAP1 was characterized later which was found to promote flowering [46,47]. Depending on the genome sequencing data of loquat (from our unpublished information), wild loquat flowering related genes of $E d F T, E d F D, E d C O$, and $E d G I$ were separated and were identified to be related to flowering regulation $[48,49]$. Most recently, we found that EjSOC1s played a vital role in promoting flowering in cultivated loquat [50]. However, the information of the upstream transcription factors participating in loquat flowering regulation is still limited.

In this work, cultivated loquat was employed as research material. The SPL homologous genes of EjSPL3, EjSPL4, EjSPL5, and EjSPL9 were isolated from "Jiefangzhong" (JFZ). The spatiotemporal expression of the EjSPLs were analyzed, and their subcellular localization were observed in tobacco leaves. In addition, the interactions between EjSPLs and other flowering proteins or the downstream flowering gene promoters were detected. Finally, because of the transform system has not been built in loquat, the genetic functions of the EjSPLs were verified in Arabidopsis thaliana. This work 
discovered the roles of EjSPL3, EjSPL4, EjSPL5, and EjSPL9 on flowering regulation, and provides further understanding of the unknown transcription factors attending reproductive growth in cultivated loquat.

\section{Results}

\subsection{Identification of SPL3, SPL4, SPL5, and SPL9 Homologs from Loquat (Eriobotrya japonica Lindl.)}

The genes of EjSPL3, EjSPL4, and EjSPL5 from the cultivated loquat "JFZ" were annotated within the genome-wide scope of "JFZ" genome (from our unpublished data), and their coding sequences were isolated. EjSPL3, EjSPL4, and EjSPL5 had 303, 570, and 576 nucleotides encoding 100, 189, and 191 amino acids, respectively (Sequence S1). Clustering analysis between the predicted amino acid sequences of the above EjSPLs and their homologous genes in other plants was conducted, the phylogenetic tree showed that EjSPL3, EjSPL4, and EjSPL5 were clustered in different branches, and the closest homologs to them were from apple or pear: EjSPL3 and MdSBPB, EjSPL4 and MdSPL1 are the closest, and EjSPL5 is closest to PbrSPL1a (Figure 1a). From the sequence alignment, there was a conserved SBP-box domain in each of them, including two zinc finger structures and a nuclear localization signal (Figure 1b). By blast alignment, EjSPL3 showed $40 \%$ and 53\% identity compared with AtSPL3 and MdSBPB; EjSPL4 had 51\% and 94\% identity compared to AtSPL4 and MdSPL1; EjSPL5 had $48 \%$ and $96 \%$ identity with AtSPL5 and PbrSPL1a, respectively. In conclusion, it is shown that the EjSPLs had higher similarity with those from apple and pear compared to other reported SPLs.
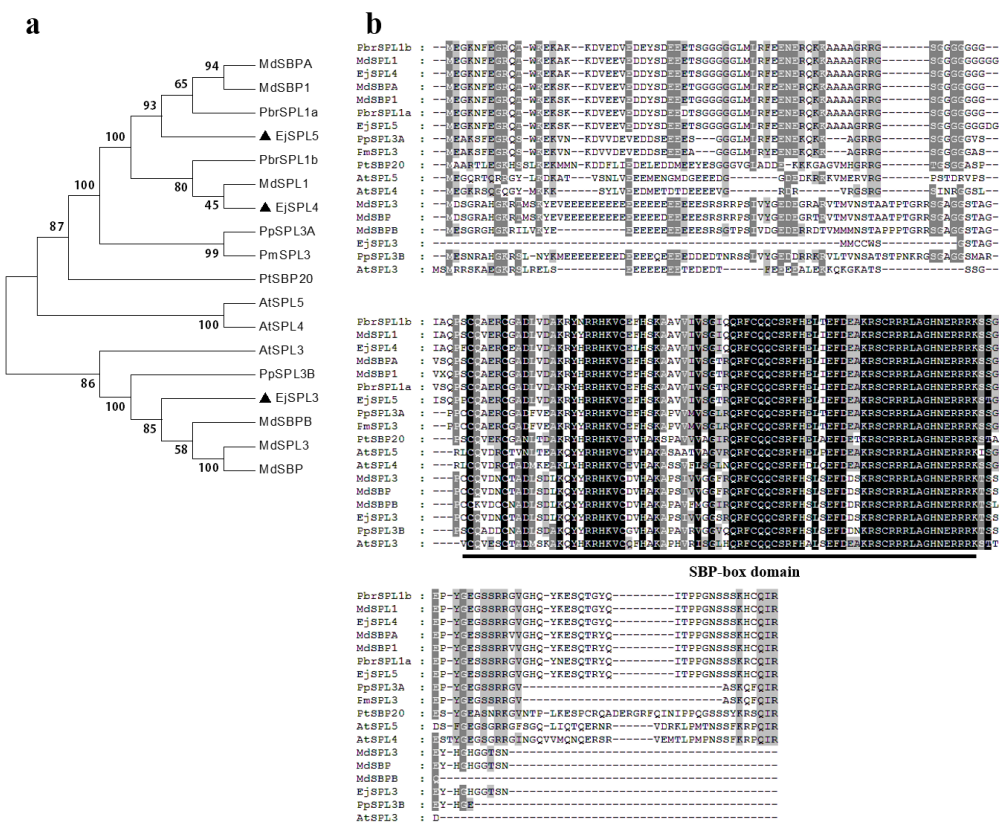

Figure 1. Sequence analysis of SPL3, SPL4, and SPL5 from various species. (a) Phylogenetic trees of SPL3, SPL4, and SPL5 in different species. Black triangles indicate loquat EjSPL3, EjSPL4, and EjSPL5. (b) Amino acid sequence alignment of SPL3, SPL4, and SPL5. Amino acids with black and gray represent $100 \%$ and $50 \%$ similarity, respectively; the underline indicates the SBP-box domain. Identical and similar amino acid residues are represented by black and grey shadows, respectively. The accession number of each gene is as follows: MdSBPA (Malus domestica, ADL36826.1), MdSBP1 (Malus domestica, XP_008386198.1), PbrSPL1a (Pyrus $\times$ bretschneideri, XP_00 9340759.1), PbrSPL1b (Pyrus $\times$ bretschneideri, XP_009356783.1), MdSPL1 (Malus domestic, XP_0083670 70.1), PpSPL3A (Prunus persica, XP_007213489.1), PmSPL3 (Prunus mume, XP_008226482.1), PtSBP20 (Populus tomentosa, AOF41322.1), AtSPL5 (AT3G15270), AtSPL4 (AT1G53160), AtSPL3 (AT2G33810), PpSPL3B (Prunus persica, XP_007212177.1), MdSBPB (Malus domestica, ADL36825.1), MdSPL3 (Malus domestica, XP_0083837 04.1), MdSBP (Malus domestica, AHC08502.1). 
Except EjSPL3, EjSPL4 and EjSPL5 genes, an EjSPL9 gene was also isolated from cultivated loquat "JFZ", and it contained 1140 nucleotides encoding 379 amino acids. The phylogenetic tree showed that EjSPL9 was closely related to SPL9 of pear and farthest from AtSPL9 and AtSPL15 (Figure 2a). From the sequence alignment, the homology of SPL9 is much higher than that of SPL3, SPL4, and SPL5 in woody plants, such as apple, pear, birch, etc., and the similarity is relatively low in herbaceous plants. In addition, they had a highly conserved SBP-box domain also (Figure 2b). By blast alignment, EjSPL9 showed $44 \%$ and $96 \%$ similarity compared with AtSPL9 and PbrSPL9, respectively.

a

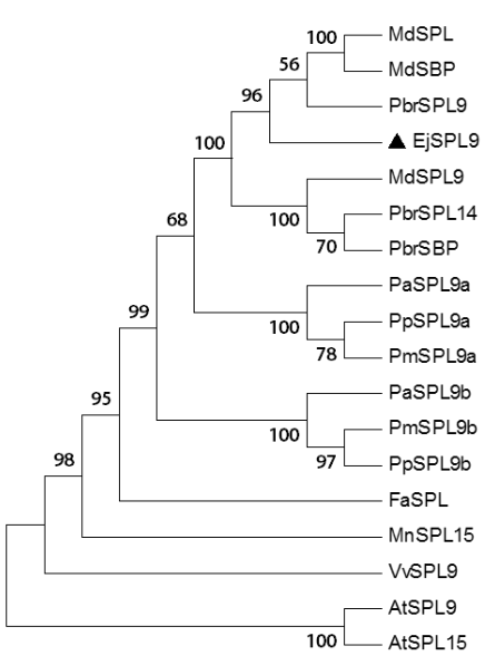

b

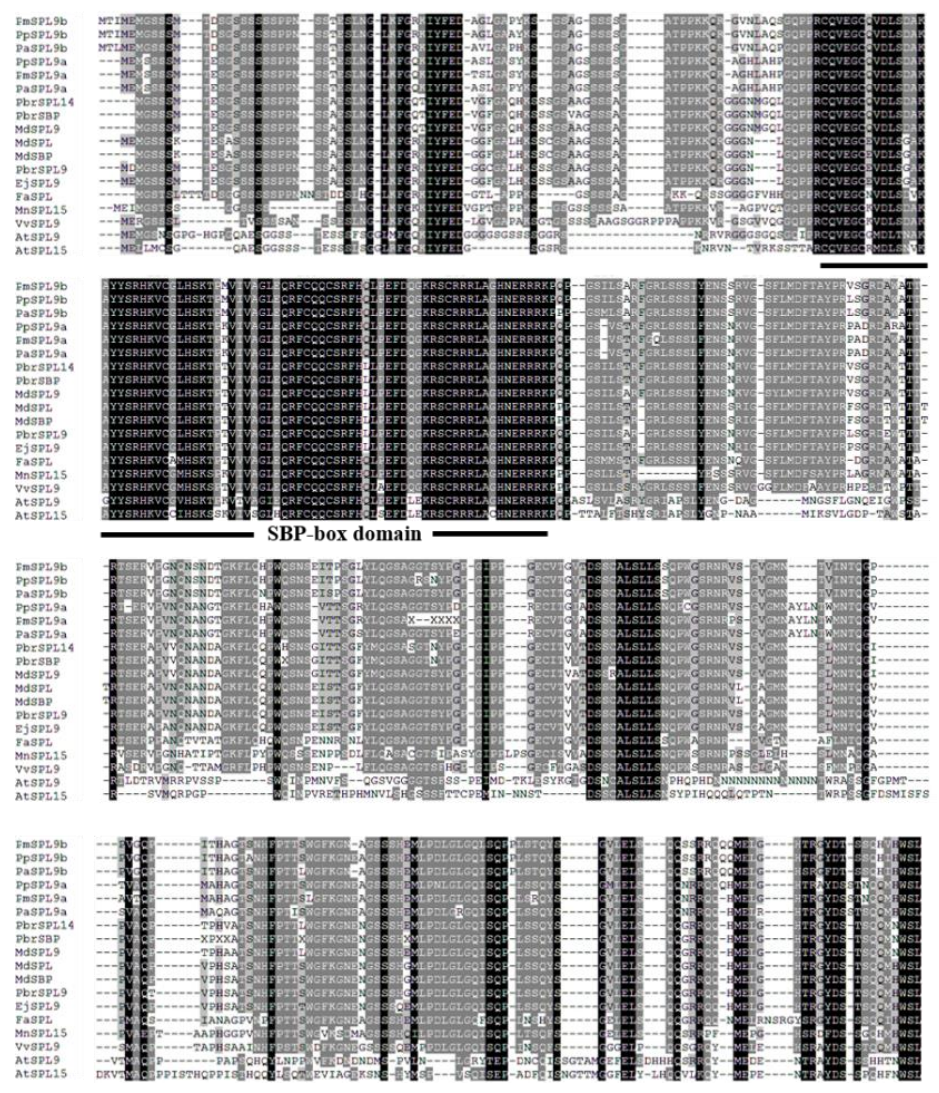

Figure 2. Sequence analysis of SPL9 from various species. (a) Phylogenetic tree of SPL9s in different species. Black triangle indicated EjSPL9. (b) Amino acid sequence alignment of SPL9s. Amino acids with black and gray represent $100 \%$ and $50 \%$ similarity, respectively; the underline indicated the SBP-box domain. Identical and similar amino acid residues are represented by black and grey shadows, respectively. The accession number of each gene is as follows: PbrSPL9 (Pyrus $\times$ bretschneideri, XP_009369782.1), MdSPL (Malus domestica, ADL36823.1), MdSBP (Malus domestica, AHC08503.1), PbrSPL14 (Pyrus $\times$ bretschneideri, XP_009376108.1), MdSPL9 (Malus domestica, XP_008392088.1), PbrSBP (Pyrus $\times$ bretschneideri, AIS93133.1), PaSPL9a (Prunus avium, XP_021830661.1), PaSPL9b (Prunus avium, XP_021825505.1), PpSPL9a (Prunus persica, XP_007203426.1), PmSPL9a (Prunus mume, XP_008240953.1), PmSPL9b (Prunus mume, XP_008240879.1), PpSPL9b (Prunus persica, XP_0072053 41.1), FaSPL (Fragaria $\times$ ananassa, AEW23126.1), VvSPL9 (Vitis vinifera, NP_001267898.1), MnSPL15 (Morus notabilis, XP_010102560.1), AtSPL9 (AT2G42200), AtSPL15 (AT3G57920).

\subsection{Analysis of EjSPLs Expression Patterns in Different Tissues}

In order to identify the role of the EjSPL3, EjSPL4, EjSPL5, and EjSPL9 in loquat flowering, we analyzed their transcription levels in the samples including leaves, buds, and flowers in different growing periods. Different tissues of the "JFZ" were sampled, including root, stem, mature leaf, leaf bud, flower bud, flower, fruit, and seed. The results showed that all EjSPLs were highly expressed 
in leaves, buds (leaf buds and flower buds), and flowers compared to other tissues. The expression level of EjSPL4, EjSPL5, and EjSPL9 in leaf buds was higher than that in flower buds, and EjSPL3 was expressed relatively higher in flower buds. However, EjSPL5 and EjSPL9 were also expressed in stem, and the transcription level of EjSPL9 was detectable in root and seed (Figure 3). The results suggested that EjSPL3, EjSPL4, EjSPL5, and EjSPL9 may be involved in the development of leaves, buds, flowers, stems, roots, and seeds.
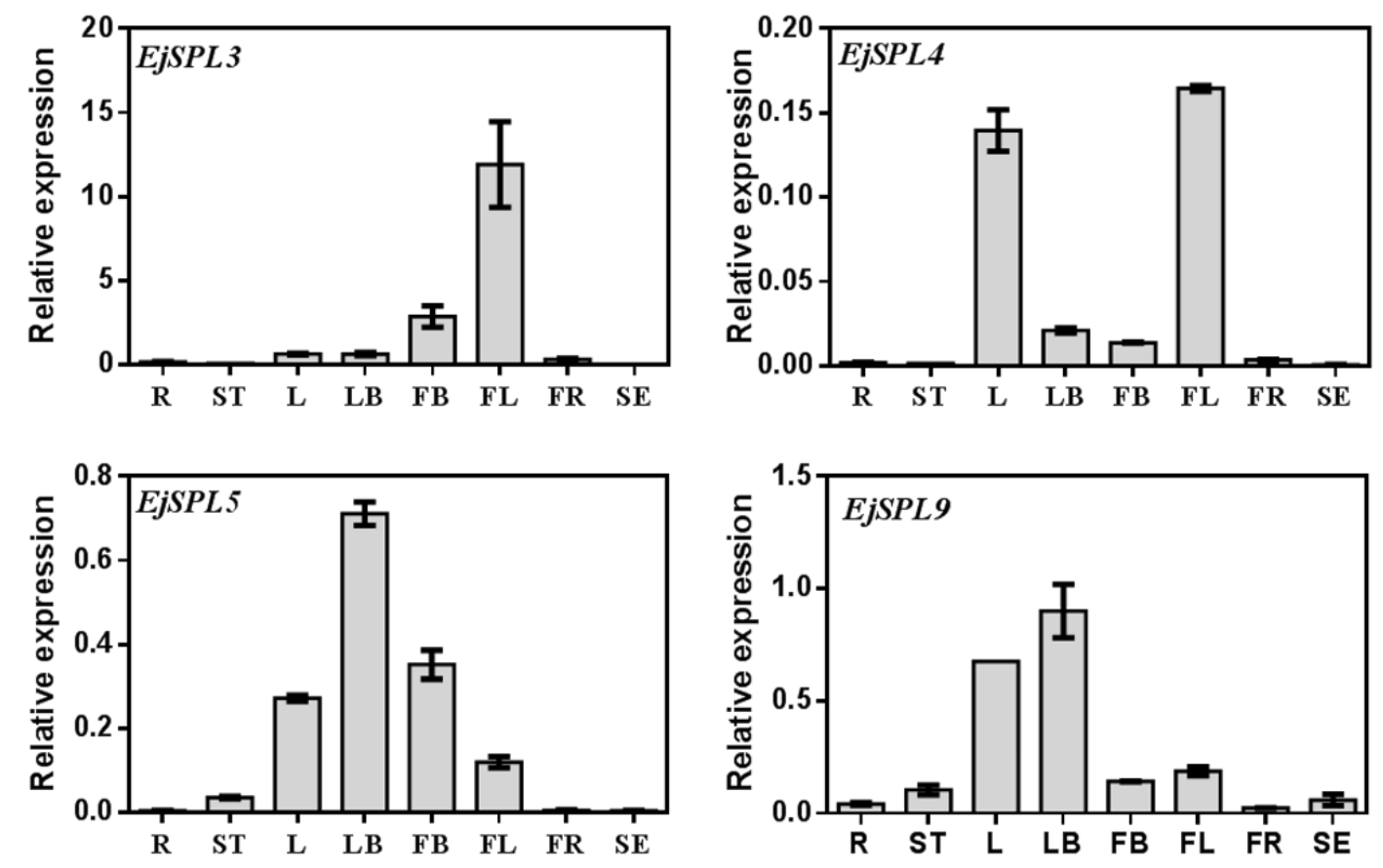

Figure 3. Relative expression levels of SPL genes in the different tissues of "JFZ". The Y-axis represents different tissues, and the X-axis represents the relative expression of the EjSPL gene of "JFZ". R-root, ST—stem, L—mature leaf (2017.4.28), LB—leaf bud (2017.04.28), FB—flower bud (2017.8.18), FL—flower (2017.12.08), FR—fruit (2017.3.17), SE—seed. Ejß-actin as an internal control. Error bars indicating SE from three biological replicates.

\subsection{Expression Pattern of EjSPLs during Growth and Development of Loquat}

To further clarify the roles of EjSPLS in loquat, the leaves, buds, and flowers of different developmental stages were sampled for detecting the EjSPLs expression levels. In our previous observation of the flower bud paraffin of "JFZ" in Guangzhou [50] the loquat flower bud differentiation happens during the period from 23 June to $7 \mathrm{July}$, and inflorescence is forming during the end of August to the beginning of September. In the leaves of different periods, the expression of EjSPL3, EjSPL4, and EjSPL5 were all in a quite low level at the beginning of the year, and followed by a significant increase till the start of flower bud differentiation (the end of June). Furthermore, the expression level of EjSPL4 and EjSPL5 gradually decreased to a low level by the end of the year, similar as that in the beginning of the year. The expression level of EjSPL9 came to a peak one month earlier than that of EjSPL4 and EjSPL5, and was kept in a relatively high level during the whole period of flower development; it just declined to a very low level after loquat finished flowering. The level of the EjSPL3 transcripts showed the first peak at the beginning of March, which was even earlier than that of EjSPL9, and also it showed another distinct peak during the early stage of flower bud development, and after that, decreased obviously to a low level by the end of the year (Figure 4a).

The expression levels of the EjSPLs in the leaves of different developmental stages (Figure 4b) showed that the expression levels of EjSPL3 and EjSPL4 were low in the young leaves and increased to a higher level as the leaves developed; after that, they began to decline to a low level again when leaves matured. EjSPL5 mRNA level was high in the young leaves and started decreasing from L3 
stage; EjSPL9 mRNA level was kept in a relatively high level in all the stages compared to other EjSPLs (Figure 4c).

a
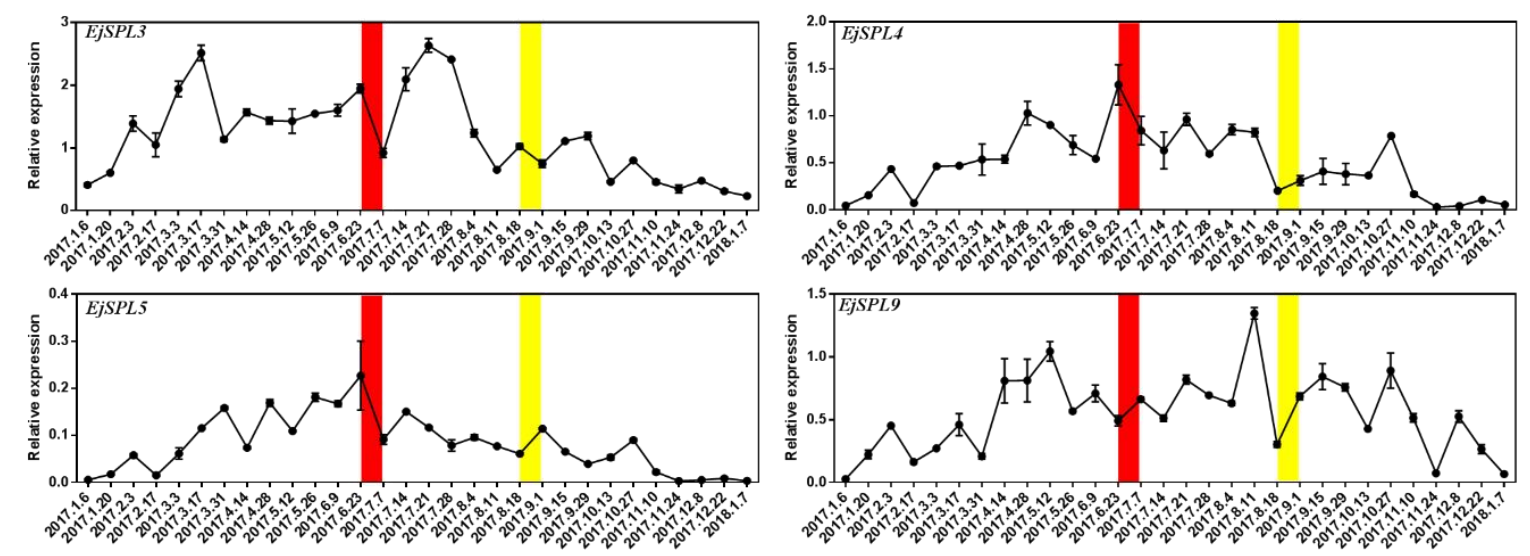

b

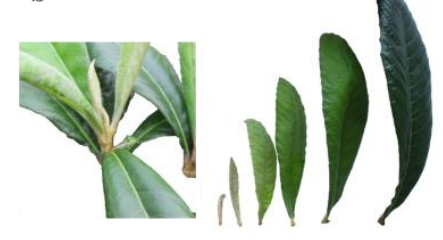

$\begin{array}{lllll}\text { L1 L2 } & \text { L3 } & \text { L4 } & \text { L5 } & \text { L6 }\end{array}$ c
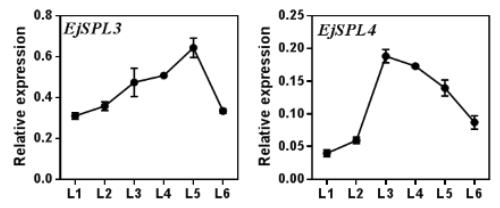

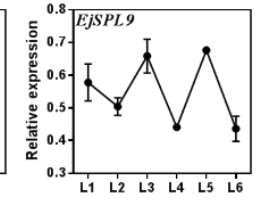

Figure 4. Relative expression levels of SPL genes in the leaves of "JFZ". (a) Expression patterns of SPL genes in the mature leaves at different periods. (b) The leaves at different developmental stages. (c) Expression patterns of SPL genes in (b). Red box and yellow box indicate the beginning of flower bud differentiation and the obvious inflorescence that can be seen, respectively. Ejß-actin as an internal control. Error bars indicating SE from three biological replicates.

AP1 is a flower meristem identity gene, located in downstream of the flowering regulation network [11], and has a high expression only in shoot apical meristem [50]; so the expression level of $A P 1$ is generally employed as a marker gene of flowering, especially for confirming the period of floral bud initiation and development. In this study, the expression level of EjAP1 showed a distinct rise from the end of June, reached the highest level in mid-August, and then declined, which is highly consistent with the period of flower bud differentiation and development in loquat (Figure 5a). In the shoot apical meristem of different periods, the expression levels of EjSPL3, EjSPL4, and EjSPL5 all came to a relatively small peak during spring, and compared with them, EjSPL9 was abundantly expressed at this period. During floral bud initiation, expression levels of all the EjSPLs started rising rapidly from 9 or 23 June, got to an obvious peak, which is ten days earlier than that of EjAP1, and then decreased immediately; therefore, it is speculated that they may be involved in the process of loquat flower bud differentiation and are upstream of EjAP1.

In addition, flower samples were collected from macroscopic flower buds to the start of fruit setting (Figure $5 b$ ). As one ABC developmental model gene, the expression level of EjAP1 was continuously increased in almost all the stages except the end of October. Importantly, EjSPL3, EjSPL4, and EjSPL5 mRNA levels showed similar trend as that of EjAP1, and they started decreasing from the end of November or the beginning of December. However, EjSPL9 showed some different or even opposite expression trends (Figure 5c). Based on these results, we speculated that EjSPL3, EjSPL4, and EjSPL5 may be involved in the growing process from the initial floral bud differentiation to the end of flowering, and EjSPL9 is hypothesized to be important for floral bud differentiation and the early stages of flower organ development. 

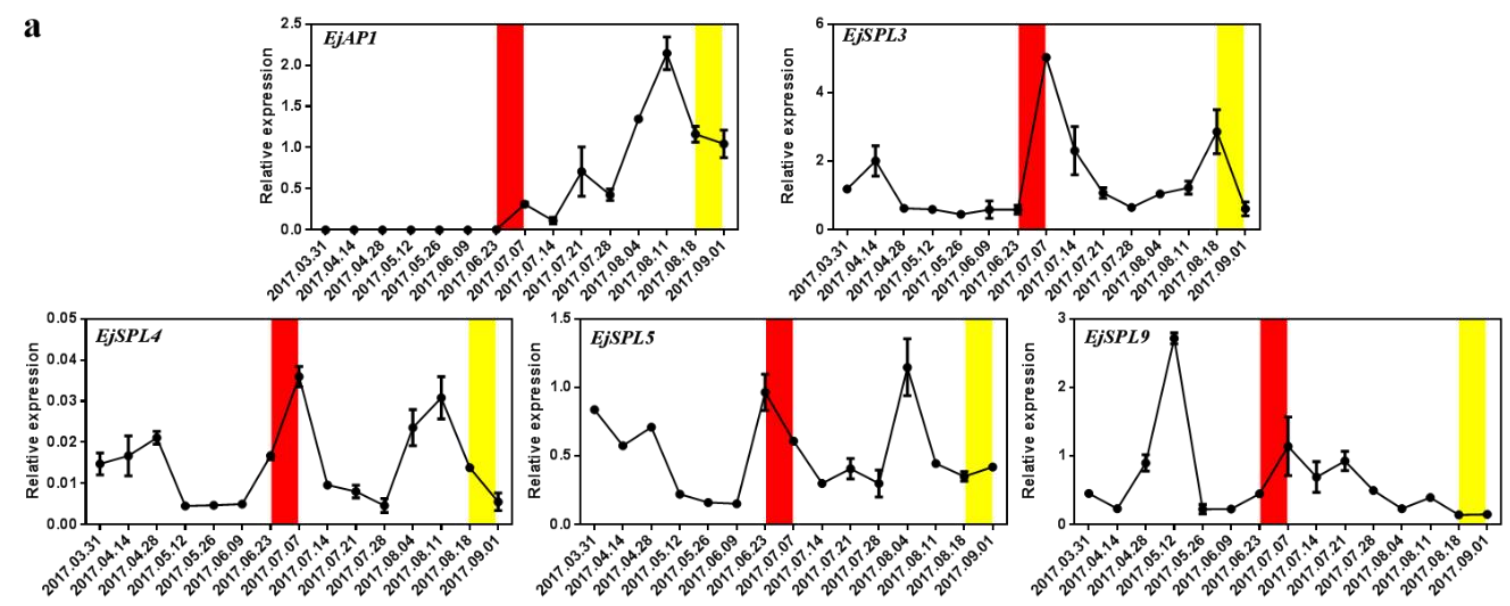

b

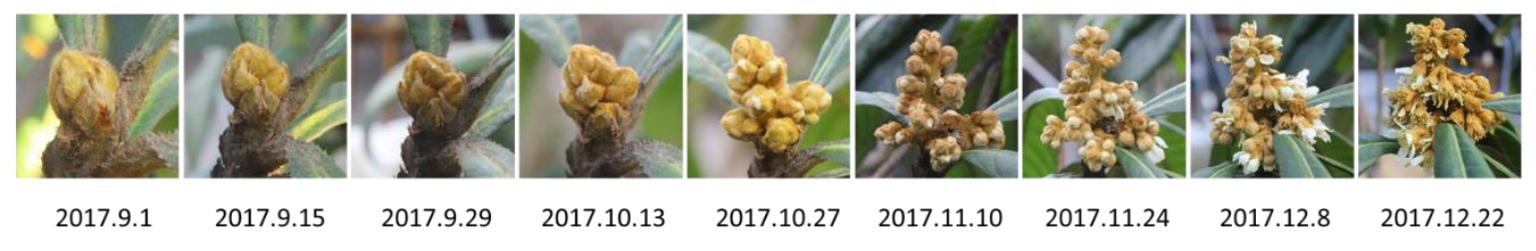

c
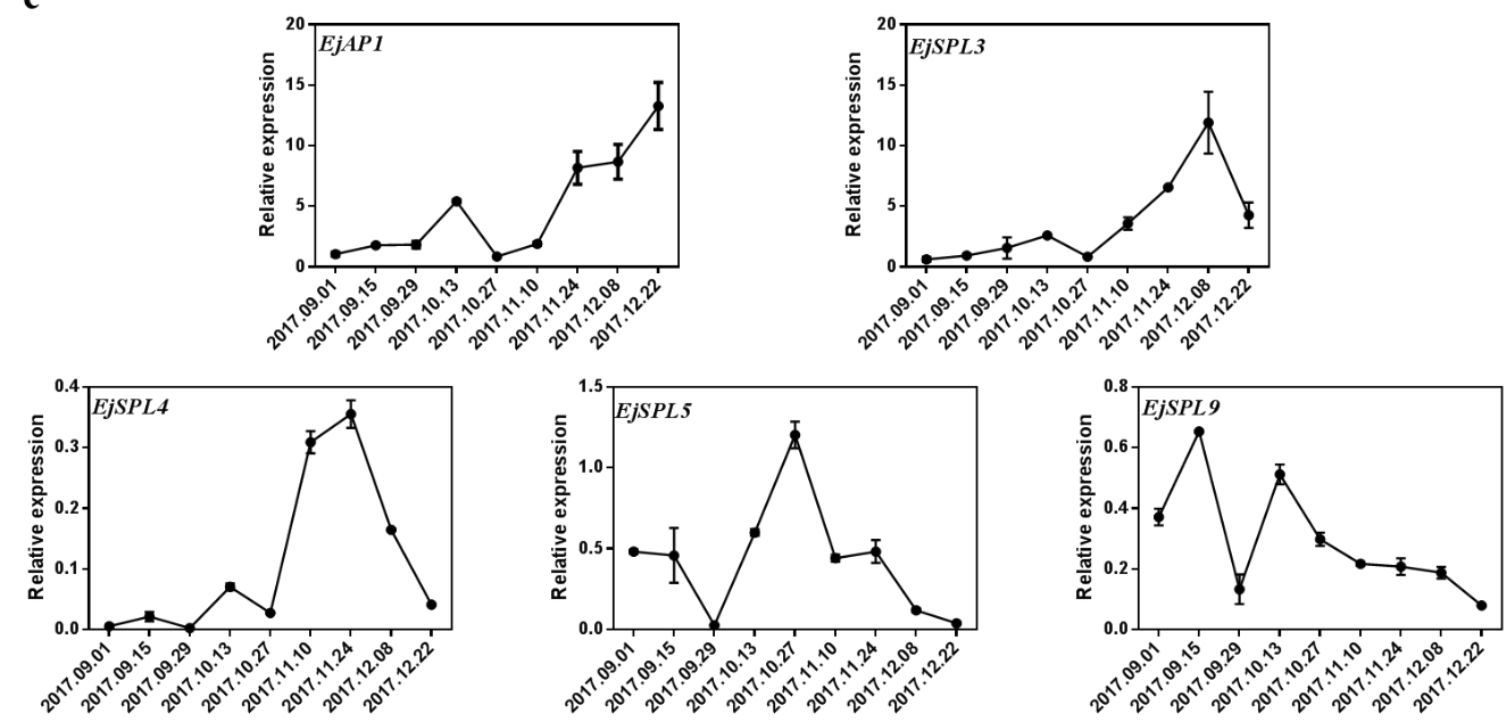

Figure 5. Relative expression levels of EjSPL genes in the buds and flowers of "JFZ" at different periods. (a) Relative expression levels of EjSPL genes in the buds at different periods. (b) The flowers in different developmental stages. (c) Relative expression levels of EjSPL genes in (b). Red box and yellow box indicated the beginning of flower bud differentiation and the obvious inflorescence that can be seen, respectively. Ejß-actin as an internal control. Error bars indicating SE from three biological replicates.

In summary, these findings suggested that EjSPL3, EjSPL4, EjSPL5, and EjSPL9 genes were possibly involved in the process of leaf development and flowering (from floral bud initiation to flower development) in loquat.

\subsection{Subcellular Localization of EjSPL3, EjSPL4, EjSPL5, and EjSPL9}

In order to verify the localization of EjSPLs-encoded proteins in cells, we constructed an EjSPLs fusion expression vector carrying 35S promoter and a green fluorescent protein (GFP)-tagged protein, and introduced them into the epidermal cells of tobacco by Agrobacterium tumefaciens infection, and then their fluorescence signals were observed. The results showed that EjSPL3, EjSPL4, and EjSPL5 
were localized in the cytoplasm and nucleus, and EjSPL9 was localized only in the nucleus (Figure 6), which suggested that EjSPL3, EjSPL4, EjSPL5, and EjSPL9 may have different functions in loquat.

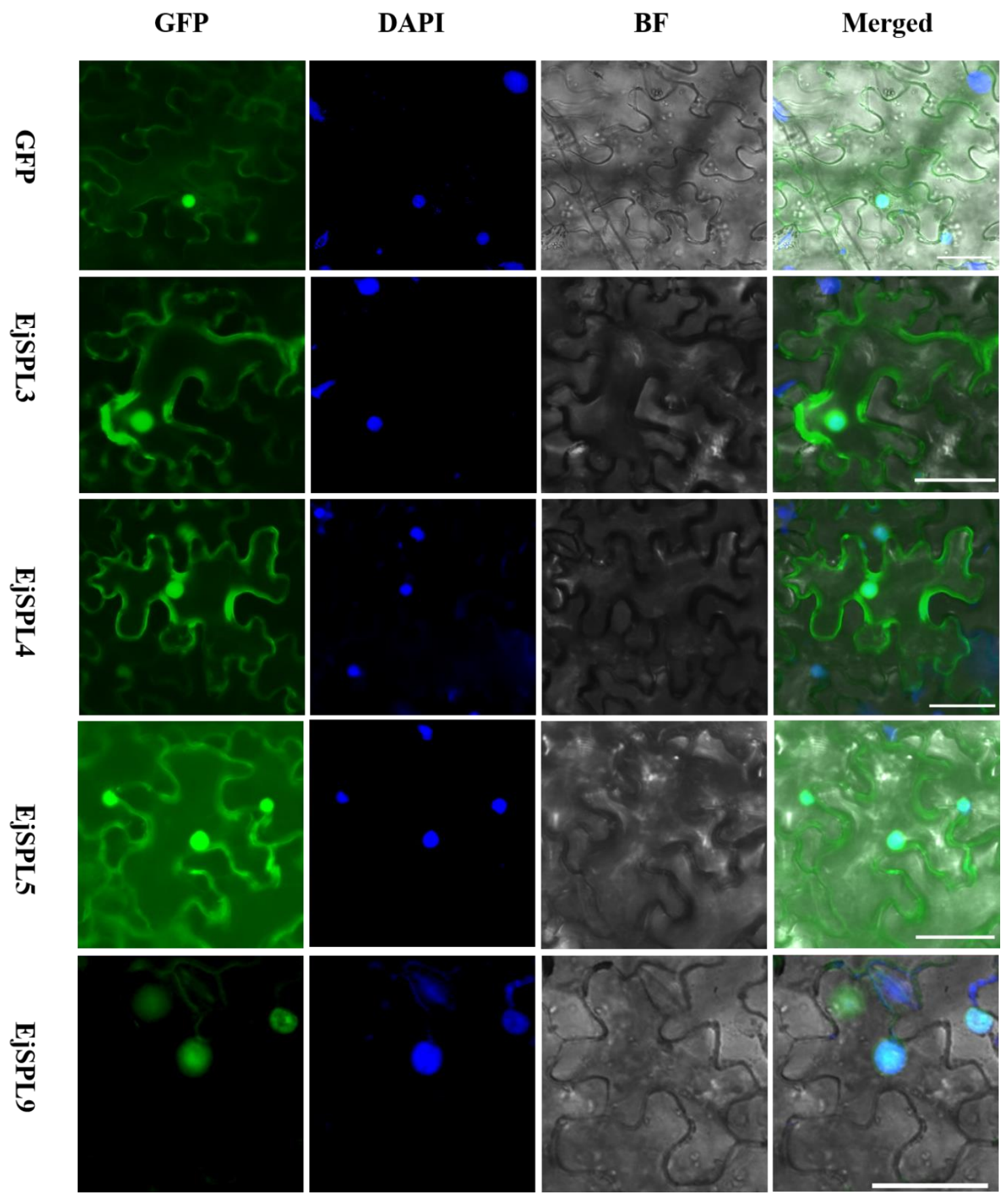

Figure 6. Subcellular localization of EjSPLs in tobacco leaves. GFP, GFP fluorescence channel; 4,6-diamidino-2-phenylindole (DAPI), DAPI fluorescence channel; BF, bright-field; Merged, merged image of GFP, DAPI, and BF. Bars $=50 \mu \mathrm{m}$.

\subsection{The regulation of EjSPL3, EjSPL4, EjSPL5, and EjSPL9 on the expression of EjLFY, EjAP1, and EjSOC1}

In the previous experiment, the expression level of EjSPLs in the apical buds showed the possibility that they may regulate the expression of the downstream flowering genes. It has been previously reported that some SPLS can directly bind to $L F Y, A P 1$, and $S O C 1$ promoters to regulate their 
transcription in Arabidopsis, and the specific binding site sequence is GTAC [35,51]. Therefore, in order to verify whether EjLFY, EjAP1, and EjSOC1 can be regulated by EjSPLs, we cloned the promoters of $E j L F Y$ s, EjAP1s, and EjSOC1s from the genomic DNA of "JFZ" loquat, respectively. The lengths of these six promoter regions are all about 2000 bp (upstream of each EjSPL genes initiation codon ATG). We used the PlaceCARE website to perform cis-acting element prediction on the promoter regions of these genes; some binding site sequences (GTAC) were found in their promoter regions (Sequence S2).

We constructed the reporter vectors, in which, CAMV35S drives renilla luciferase (REN), and the EjLFY-1pro, EjLFY-2pro, EjSOC1-1pro, EjSOC1-2pro, EjAP1-1pro, and EjAP1-2pro drive firefly luciferase (LUC). EjSPL3, EjSPL4, EjSPL5, and EjSPL9 were constructed into the effector vector driven by CAMV35S (Figure 7a). The two vectors were co-transformed into tobacco by transient expression by different combinations to analyze whether EjSPL3, EjSPL4, EjSPL5, and EjSPL9 could transcriptionally activate the downstream genes promoters. From the luciferase signal, it was showed that EjSPL3, EjSPL4, EjSPL5, and EjSPL9 obviously activated the expression of LUC, which was driven by the promoters of EjLFY-1 and EjSOC1-1; and EjSPL4, EjSPL5, and EjSPL9 remarkably activated the expression of LUC driven by the EjAP1-1 promoter compared to the control (Figure 7b).

\section{$\mathbf{a}$}
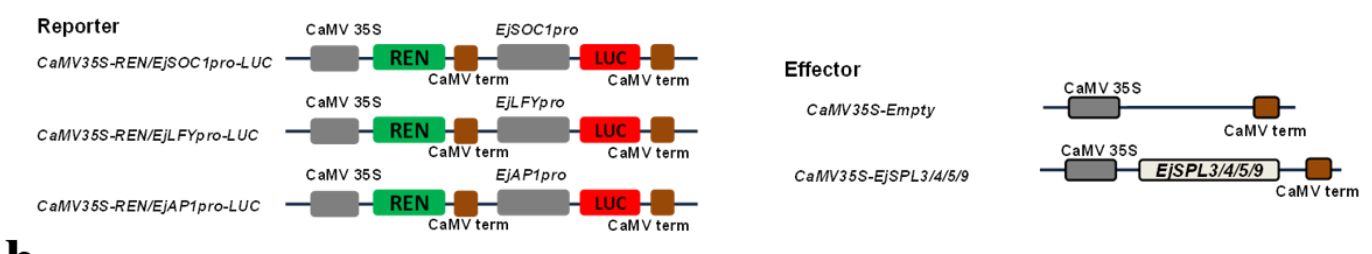

b
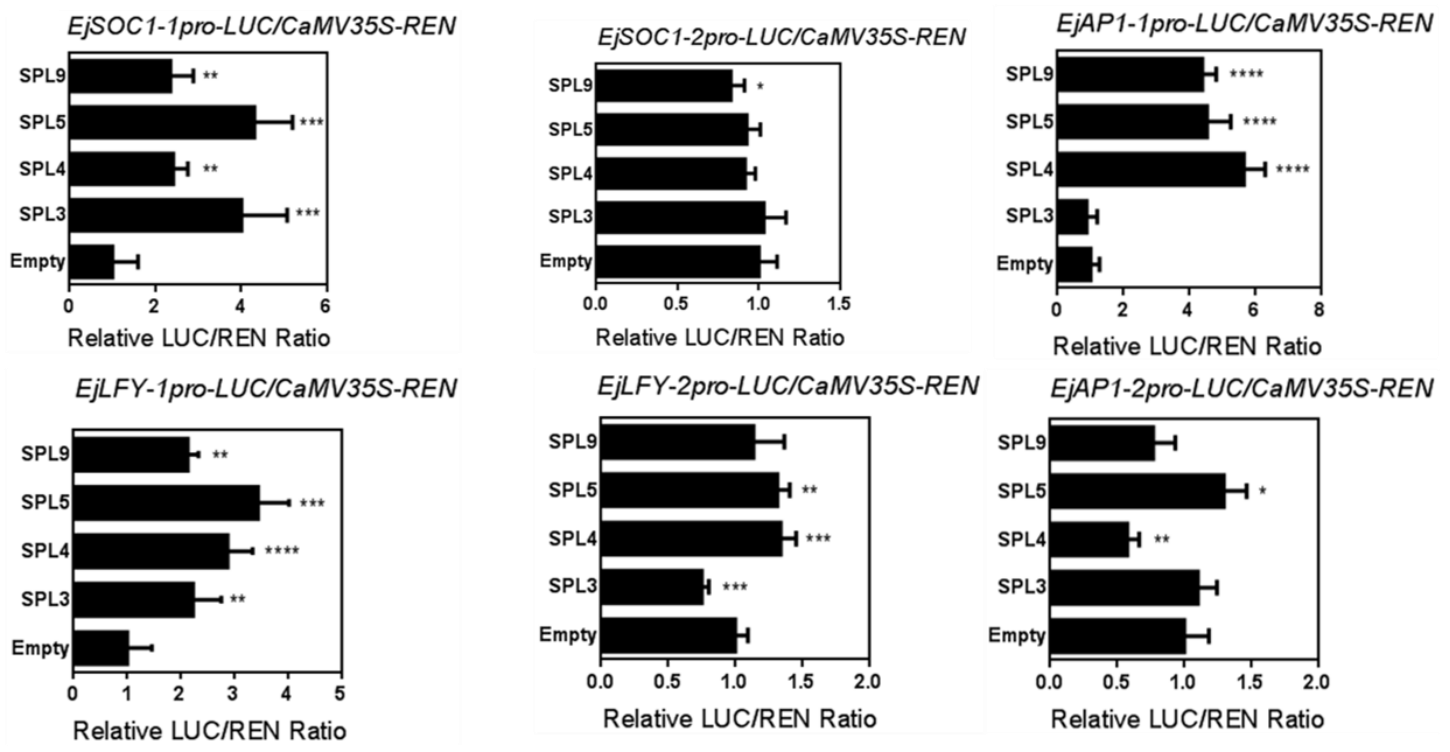

Figure 7. The activation of EjSPL3, EjSPL4, EjSPL5 and EjSPL9 on the expression of EjLFY, EjAP1, and EjSOC1. The activation effect was detected using dual luciferase system. (a) Vectors construction. (b) EjSPL3, EjSPL4, EjSPL5, and EjSPL9 activated the EjLFY, EjAP1, and EjSOC1 promoters. Error bars indicating SE from at least six biological replicates. The asterisk indicates a significant difference compared to the empty effector (Student's $t$-test, ${ }^{*} p<0.01,{ }^{* *} p<0.001$, ${ }^{* * *} p<0.0001,{ }^{* * *} p<0.00001$ ).

\subsection{EjSPL3, EjSPL4, EjSPL5, and EjSPL9 Promote Flowering in Arabidopsis}

To investigate the genetic functions of EjSPL3, EjSPL4, EjSPL5, and EjSPL9, overexpression vectors containing full length coding sequences of the four EjSPL genes were constructed and transformed into wild-type Arabidopsis thaliana (Col-0). The homozygous transgenic lines were screened out, and the flowering time and the expression of downstream flowering genes were analyzed in these 
transgenic lines. All transgenic lines with different genes showed early flowering phenotype compared to wild-type Col-0 (Figure 8a). Under the same growing condition, Col-0 had about 12 rosette leaves when flowering, while the transgenic lines with 35S: EjSPL3 and 35S: EjSPL5 had only seven to eight rosette leaves; 35S: EjSPL4 had nine to ten rosette leaves; 35S: EjSPL9 had 10 to 11 rosette leaves (Figure 8b). The expression levels of the related flowering genes including AtAP1, AtLFY, and AtSOC1, showed different degrees of improvement (Figure S1). There was no obvious different morphological characteristic of the above transgenic lines observed compared to that of Col-0, such as flower organs, leaf shapes, siliques, and cauline leaves. From the above results, EjSPL3, EjSPL4, EjSPL5, and EjSPL9 all have the function of promoting flowering in Arabidopsis.

a

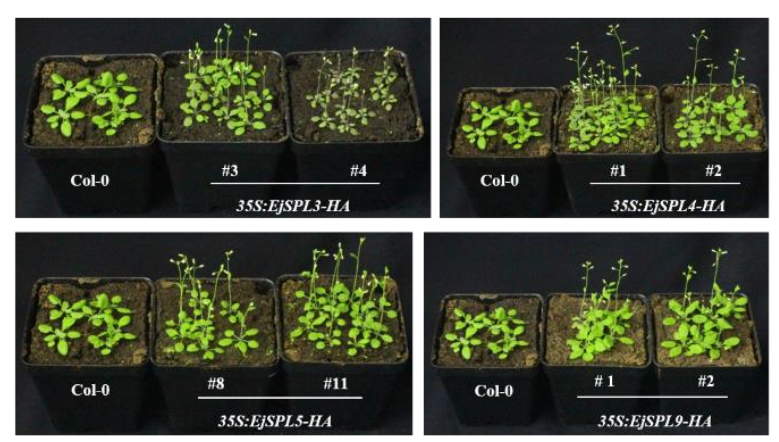

b

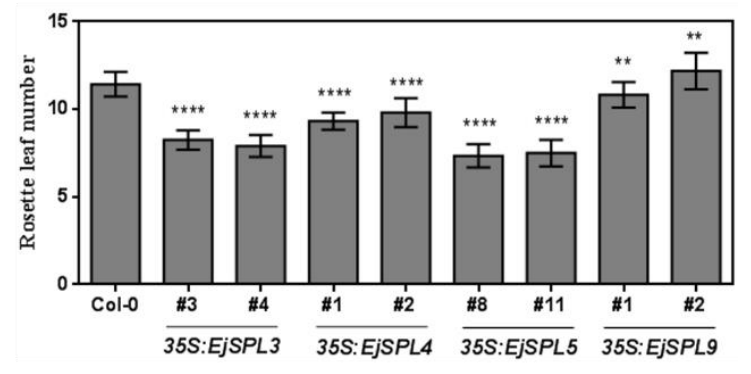

Figure 8. Overexpression of EjSPL3, EjSPL4, EjSPL5, and EjSPL9 in Arabidopsis promotes early flowering. (a) Phenotype of overexpression transgenic lines and wild Col-0. (b) The rosette leaf numbers of overexpression transgenic lines and Col-0 in (a). Error bars indicating SE from three biological replicates. The asterisk indicates a significant difference compared to the Col-0 $\left(n \geq 15\right.$, Student's $t$-test, ${ }^{* *} p<0.01$, **** $p<0.00001)$.

\section{Discussion}

To date, remarkable achievements have been made in the research of flowering regulation mechanism in plants, including photoperiod pathway, gibberellin pathway, autonomous pathway, aging pathway, vernalization pathway, ambient temperature pathway, and so on, which integrate each other to form a huge and complex regulatory network [52]. Among the above pathways, SPL transcription factors play a very important role in the control of flowering time. Our results show that all members of the SPL gene family contain the SBP-box domain, which was conserved in a certain degree in different species (Figures 1 and 2). In this study, it was suggested that EjSPL3, EjSPL4, EjSPL5, and EjSPL9 are involved in the regulation of flowering time in loquat and are highly conserved with their homologous genes. Differential expression levels and subcellular localization implied that they may have different patterns in regulating loquat growth and development.

Functional verification of SPL3, SPL4, SPL5, and SPL9 homologous genes in other plants has also been reported. For example, overexpression of SPL3 in Arabidopsis wild-type results in early flowering, and knocking out SPL3, SPL4, SPL5, and SPL9 delays flowering time and produces more rosette leaves [13]. MdSPL3 of apple encodes 189 amino acids and is localized in the nucleus; overexpressing it in Arabidopsis wild-type turns into fewer trichomes and promotes morphological differentiation, the MdSPL3 to be highly expressed in the rosette leaves and stem of transgenic Arabidopsis, and finally significantly improves the expression of downstream genes (AtFT, AtSOC1, AtLFY, AtAP1, AtFUL) [53]. In citrus, overexpression of CiSPL5 removed the binding site of miR156 in the 3'UTR, to Arabidopsis wild-type, leading to early flowering and fewer small rosette leaves [54]. PtSPL9 is expressed in various organs, with the highest expression in stems, and showed a nuclear localization [55]. In grape, VpSBP11 (a homolog of AtSPL3/4/5) encoded 170 amino acids, contained a highly conserved SBP domain, and its protein was localized in the nucleus with the transcriptional activity; overexpressing it (removed $3^{\prime}$ UTR, the binding site of miR156) into the Arabidopsis wild-type advanced flowering, and promoted 
the expression of $F U L, A P 1, L F Y$, and some other genes; and also, the phenotypic characteristics in transgenic plants were transformed [36]. Combined with our results, it is shown that the SPL genes are both functionally conserved and functionally differentiated during plant development.

The regulatory network of SPL is extremely complicated, and to date, the model plant Arabidopsis is the most thoroughly studied. Most of the SPL genes in Arabidopsis are targeted by miR156 and miR157 [14,56,57], and positively regulate downstream flowering genes directly, such as SOC1, LFY, $A P 1, F U L$, and so on $[35,51]$. This regulation pattern has also been verified in other species $[28,53,58,59]$. In this study, we found some SPL binding sites (GTAC) in the promoter regions of EjLFY, EjSOC1, and EjAP1 (Sequence S2); and the dual-luciferase reporter assays verified that EjSPLs activate their expression, in which, EjSPL3, EjSPL4, EjSPL5, and EjSPL9 effectively activated EjLFY-1 and EjSOC1-1 expression; and EjSPL4, EjSPL5, and EjSPL9 significantly improve the expression of EjAP1-1 (Figure 7). In addition, we found that the expression levels of the floral meristem identity genes AtAP1 and AtLFY were significantly different among different transgenic lines (Figure S1). These results suggested that the SPL genes in loquat may be coordinated with each other to act on different downstream genes to jointly regulate flowering.

There are also some novel regulatory models found in other plants. SISBP3 and SISBP15 of tomato are regulated by the activity of PROCERA/DELLA, which can activate the SINGLE FLOWER TRUSS (SFT) gene in the leaves and the AP1/MC in the shoot apex to promote flowering [60]. In the Arabidopsis thaliana, SODIUM POTASSUIM ROOT DEFECTIVE1 (NaKR1) was found to respond to the changes in potassium, and it could regulate the expression of $F T$ through the miR156-SPL3 module, which also affected the transport of FT protein in the phloem [61]. The nuclear factor CmNF-YB8 is a direct upstream gene of cmo-miR156, which can directly bind to its promoter region. Silencing this gene led to down-regulation of cmo-miR156 and up-regulation of CmSPL3, CmSPL5, and CmSPL9 [58]. Interestingly, EjFT2 showed involvement in the process of flower bud differentiation in loquat [62]. In order to ascertain whether there is a similar pattern of flowering regulation in the EjSPL genes in loquat, we need more in-depth research.

\section{Materials and Methods}

\subsection{Plant Materials and Growth Conditions}

Cultivated loquat (Eriobotrya japonica Lindl.) was used in this study. Adult loquat trees were grown under natural conditions in the loquat germplasm resource preservation garden, South China Agricultural University, Guangzhou, China. The wild-type of Arabidopsis, Arabidopsis thaliana Columbia-0 (Col-0), was used for gene transformation. Nicotiana benthamiana was cultivated for the assays of transient expression. The seeds of the Arabidopsis Col-0 and tobacco used in this study were provided by Xingliang Hou. Both of Arabidopsis and tobacco were grown under long-day conditions (16 h light/ $8 \mathrm{~h}$ dark) at $22{ }^{\circ} \mathrm{C}$ in a controlled environment room. Samples (loquat tissues and Arabidopsis plants) for qRT-PCR were immediately frozen in liquid nitrogen and stored in an ultra-low temperature refrigerator at $-80^{\circ} \mathrm{C}$ until use.

\subsection{Gene Cloning and Sequence Analysis}

The coding region of EjSPL3, EjSPL4, EjSPL5, and EjSPL9 were amplified through PCR using PrimeSTAR ${ }^{\circledR}$ Max DNA Polymerase (TaKaRa, shiga, Japan) with specific primers (Table S1). The PCR products were connected with pGEM-T vector (Promega, Wisconsin, USA). Then the cloned products were sequenced and blasted with the other homologous sequences in the NCBI (https: //www.ncbi.nlm.nih.gov/). DNAMAN 6.0, ClustalX 2.0.12, GeneDoc 2.7, and MEGA 6 software were employed for the amino acid sequences alignment and the construction of the phylogenetic tree of $S P L$ proteins, respectively. 


\subsection{Gene Expression Analysis with $q R T-P C R$}

The total RNA of loquat and Arabidopsis was extracted by EASYspin Plus plant RNA extraction kit (Aid lab, Beijing, China), and the cDNA was synthesized using PrimeScript ${ }^{\mathrm{TM}}$ RT reagent Kit with gDNA Eraser (TaKaRa, shiga, Japan). Quantitative real-time PCR (qPCR) was performed using iTaq ${ }^{\mathrm{TM}}$ universal SYBR Green Supermix (Bio-Rad, Hercules, CA, USA) in the LightCycler 480 (Roche, Basel Switzerland). Ejß-actin [63] and TUB2 (AT5G62690) were amplified as the internal control genes for normalization in loquat and Arabidopsis thaliana, respectively. Three technical and biological replicates were applied and data were analyzed with previous methods [64]. Semi-quantitative RT-PCR was employed for detecting the expression of exogenous gene in the overexpression lines of Arabidopsis.

\subsection{Subcellular Localization Analysis}

The full length coding sequences of EjSPL3, EjSPL4, EjSPL5, and EjSPL9 without stop codon were separately cloned into the pGreen-35S-GFP vector [65]. These pGreen-35S-EjSPL-GFP or negative control vectors were transformed into the Agrobacterium tumefaciens strain GV3101::psoup by heat induction, and the transformed Agrobacterium cells were cultured and injected into epidermic cells of tobacco (Nicotiana benthamiana) leaves for transient expression following the previously described methods [66]. After about $48 \mathrm{~h}$ of injection, GFP signal was observed using the fluorescence microscope (Observer.D1, Zeiss, Jena, Germany).

\subsection{Arabidopsis Transformation}

The full length coding sequences of EjSPL3, EjSPL4, EjSPL5, and EjSPL9 were alone cloned into pGreen-35S-6HA vector [67] to generate the overexpressed EjSPLs. Then, these recombined vectors were transformed into Arabidopsis Col-0 by the floral dip through Agrobacterium-mediated method [68]. Finally, T3 homozygous transgenic lines were screened using Basta.

\subsection{Promoter Cloning and Analysis}

The promoters of EjLFY, EjAP1 and EjSOC1 were cloned from the DNA of "JFZ" using PrimeSTAR ${ }^{\circledR}$ Max DNA Polymerase (TaKaRa) with specific primers. Putative cis-acing elements in each promoter region were found in the PlantCARE database (http://bioinformatics.psb.ugent.be/webtools/plantcare/ $\mathrm{html} /)$.

\subsection{Dual-luciferase Reporter Assays}

For the analysis of binding activity of EjSPL3, EjSPL4, EjSPL5, and EjSPL9 to the promoters of EjLFY, EjAP1, and EjSOC1, the above promoters were cloned into pGreenII 0800-LUC double-reporter vector, firefly luciferase (LUC), and Renilla luciferase (REN), and the full length coding sequences of EjSPL3, EjSPL4, EjSPL5, and EjSPL9 without stop codon were cloned into pGreenII 62-SK effector vector [69].

The recombined effectors and reporters were co-transformed into tobacco leaves with different groups by Agrobacterium-mediated protocol, as described above. After about $48 \mathrm{~h}$ of transformation, we quantified the activity of LUC and REN using the dual luciferase assay kit (Promega) with appropriate modification [70] was quantified on the Luminoskan Ascent Microplate Luminometer (Thermo Fisher Scientific). The binding activity was calculated by the LUC to REN ratio. Each assay was made by at least six biological duplicates.

\subsection{Data Analysis}

All data analyses were performed by Excel and GraphPad Prism 6.01. Significance of differences between data was evaluated by Student's $t$ test. 


\section{Conclusions}

In summary, the Eriobotrya japonica transcription factors EjSPL3, EjSPL4, EjSPL5, and EjSPL9 are speculated to participate in flower bud differentiation and flower organ development by activating the expression of EjSOC1, EjLFY, or EjAP1.

Supplementary Materials: Supplementary materials can be found at http://www.mdpi.com/1422-0067/21/1/248/s1. Table S1; Sequence S1; Sequence S2; Figure S1.

Author Contributions: Y.J. (Yuanyuan Jiang), J.P., S.L., and Y.G. designed the research. J.P. and Y.J. (Yuanyuan Jiang) mainly performed the research. M.W., W.S., X.G., Y.J. (Yi Jing) and X.Y. finished some parts of the experiments. J.P. wrote the manuscript. S.L. and Y.G. revised and approved the manuscript. All authors have read and agreed to the published version of the manuscript.

Funding: This study was financially supported by the Natural Science Foundation of Guangdong Province (2017A030313129), the Earmarked Fund for Guangdong Modern Agro-industry Technology Research System (2018LM1128), the Ministry of Agriculture Industry Technology Special Project (201003073), Key Realm R\&D Program of GuangDong Provide (2018B020202011), and Promotion and application of high-efficiency utilization technology of livestock and poultry wastewater in the cultivation of loquat cultivars (2018A0115).

Acknowledgments: We thank Xingliang Hou for providing pGreen-35S-6HA and pGreen-35S-GFP plasmids, Arabidopsis Col-0, and tobacco seeds. We wish to thank American Journal Experts for their language editing services for this manuscript.

Conflicts of Interest: The authors declare no conflict of interest. The funders had no role in the design of the study; in the collection, analyses, or interpretation of data; in the writing of the manuscript, or in the decision to publish the results.

\section{References}

1. Blazquez, M.A.; Weigel, D. Integration of floral inductive signals in Arabidopsis. Nature 2000, 404, 889-892. [CrossRef] [PubMed]

2. Amasino, R.M.; Michaels, S.D. The Timing of Flowering. Plant Physiol. 2010, 154, 516-520. [CrossRef] [PubMed]

3. Fornara, F.; de Montaigu, A.; Coupland, G. SnapShot: Control of flowering in Arabidopsis. Cell 2010, 141, 550. [CrossRef] [PubMed]

4. Srikanth, A.; Schmid, M. Regulation of flowering time: All roads lead to Rome. Cell. Mol. Life Sci. 2011, 68, 2013-2037. [CrossRef] [PubMed]

5. Yu, S.; Cao, L.; Zhou, C.-M.; Zhang, T.-Q.; Lian, H.; Sun, Y.; Wu, J.; Huang, J.; Wang, G.; Wang, J.-W. Sugar is an endogenous cue for juvenile-to-adult phase transition in plants. Elife 2013, 2. [CrossRef]

6. Zuo, Z.; Liu, H.; Liu, B.; Liu, X.; Lin, C. Blue Light-Dependent Interaction of CRY2 with SPA1 Regulates COP1 activity and Floral Initiation in Arabidopsis. Curr. Biol. 2011, 21, 841-847. [CrossRef]

7. Putterill, J.; Laurie, R.; Macknight, R. It's time to flower: The genetic control of flowering time. Bioessays 2004, 26, 363-373. [CrossRef]

8. Wellmer, F; Riechmann, J.L. Gene networks controlling the initiation of flower development. Trends Genet. 2010, 26, 519-527. [CrossRef]

9. Wang, J.-W. Regulation of flowering time by the miR156-mediated age pathway. J. Exp. Bot. 2014, 65, 4723-4730. [CrossRef]

10. Kardailsky, I.; Shukla, V.K.; Ahn, J.H.; Dagenais, N.; Christensen, S.K.; Nguyen, J.T.; Chory, J.; Harrison, M.J.; Weigel, D. Activation tagging of the floral inducer FT. Science 1999, 286, 1962-1965. [CrossRef]

11. Ferrandiz, C.; Gu, Q.; Martienssen, R.; Yanofsky, M.F. Redundant regulation of meristem identity and plant architecture by FRUITFULL, APETALA1 and CAULIFLOWER. Development 2000, 127, 725-734. [PubMed]

12. Lee, J.-H.; Park, C.-M. Integration of photoperiod and cold temperature signals into flowering genetic pathways in Arabidopsis. Plant Signal. Behav. 2015, 10, e1089373. [CrossRef] [PubMed]

13. Xu, M.; Hu, T.; Zhao, J.; Park, M.-Y.; Earley, K.W.; Wu, G.; Yang, L.; Poethig, R.S. Developmental Functions of miR156-Regulated SQUAMOSA PROMOTER BINDING PROTEIN-LIKE (SPL) Genes in Arabidopsis thaliana. PLoS Genet. 2016, 12, e1006263. [CrossRef] [PubMed] 
14. He, J.; Xu, M.; Willmann, M.R.; McCormick, K.; Hu, T.; Yang, L.; Starker, C.G.; Voytas, D.F.; Meyers, B.C.; Poethig, R.S. Threshold-dependent repression of SPL gene expression by miR156/miR157 controls vegetative phase change in Arabidopsis thaliana. PLoS Genet. 2018, 14. [CrossRef]

15. Jones-Rhoades, M.W.; Bartel, D.P.; Bartel, B. MicroRNAs and their regulatory roles in plants. Annu. Rev. Plant Biol. 2006, 57, 19-53. [CrossRef]

16. Chuck, G.; O'Connor, D. Small RNAs going the distance during plant development. Curr. Opin. Plant Biol. 2010, 13, 40-45. [CrossRef]

17. Klein, J.; Saedler, H.; Huijser, P. A new family of DNA binding proteins includes putative transcriptional regulators of the Antirrhinum majus floral meristem identity gene SQUAMOSA. Mol. Gen. Genet. 1996, 250, 7-16. [CrossRef]

18. Cardon, G.H.; Hoehmann, S.; Nettesheim, K.; Saedler, H.; Huijser, P. Functional analysis of the Arabidopsis thaliana SBP-box gene SPL3: A novel gene involved in the floral transition. Plant J. 1997, 12, 367-377. [CrossRef]

19. Yang, Z.; Wang, X.; Gu, S.; Hu, Z.; Xu, H.; Xu, C. Comparative study of SBP-box gene family in Arabidopsis and rice. Gene 2008, 407, 1-11. [CrossRef]

20. Tripathi, R.K.; Bregitzer, P.; Singh, J. Genome-wide analysis of the SPL/miR156 module and its interaction with the AP2/miR172 unit in barley. Sci. Rep. 2018, 8, 7085. [CrossRef]

21. Kropat, J.; Tottey, S.; Birkenbihl, R.P.; Depege, N.; Huijser, P.; Merchant, S. A regulator of nutritional copper signaling in Chlamydomonas is an SBP domain protein that recognizes the GTAC core of copper response element. Proc. Natl. Acad. Sci. USA 2005, 102, 18730-18735. [CrossRef] [PubMed]

22. Riese, M.; Hoehmann, S.; Saedler, H.; Muenster, T.; Huijser, P. Comparative analysis of the SBP-box gene families in P-patens and seed plants. Gene 2007, 401, 28-37. [CrossRef] [PubMed]

23. Salinas, M.; Xing, S.; Hoehmann, S.; Berndtgen, R.; Huijser, P. Genomic organization, phylogenetic comparison and differential expression of the SBP-box family of transcription factors in tomato. Planta 2012, 235, 1171-1184. [CrossRef] [PubMed]

24. Zhang, L.; Wu, B.; Zhao, D.; Li, C.; Shao, F.; Lu, S. Genome-wide analysis and molecular dissection of the SPL gene family in Salvia miltiorrhiza. J. Integr. Plant Biol. 2014, 56, 38-50. [CrossRef]

25. Xie, K.; Wu, C.; Xiong, L. Genomic organization, differential expression, and interaction of SQUAMOSA promoter-binding-like transcription factors and microRNA156 in rice. Plant Physiol. 2006, 142, 280-293. [CrossRef]

26. Lannenpaa, M.; Janonen, I.; Holtta-Vuori, M.; Gardemeister, M.; Porali, I.; Sopanen, T. A new SBP-box gene BpSPL1 in silver birch (Betula pendula). Physiol. Plant 2004, 120, 491-500. [CrossRef]

27. Li, X.-Y.; Lin, E.-P.; Huang, H.-H.; Niu, M.-Y.; Tong, Z.-K.; Zhang, J.-H. Molecular Characterization of SQUAMOSA PROMOTER BINDING PROTEIN-LIKE (SPL) Gene Family in Betula luminifera. Front. Plant Sci. 2018, 9, 608. [CrossRef]

28. Cai, C.; Guo, W.; Zhang, B. Genome-wide identification and characterization of SPL transcription factor family and their evolution and expression profiling analysis in cotton. Sci. Rep. 2018, 8, 762. [CrossRef]

29. Hou, H.; Li, J.; Gao, M.; Singer, S.D.; Wang, H.; Mao, L.; Fei, Z.; Wang, X. Genomic Organization, Phylogenetic Comparison and Differential Expression of the SBP-Box Family Genes in Grape. PLoS ONE 2013, 8, e59358. [CrossRef]

30. Li, J.; Hou, H.; Li, X.; Xiang, J.; Yin, X.; Gao, H.; Zheng, Y.; Bassett, C.L.; Wang, X. Genome-wide identification and analysis of the SBP-box family genes in apple (Malus $x$ domestica Borkh.). Plant Physiol. Biochem. 2013, 70, 100-114. [CrossRef]

31. Zhang, W.; Li, B.; Yu, B. Genome-wide identification, phylogeny and expression analysis of the SBP-box gene family in maize (Zea mays). J. Integr. Agric. 2016, 15, 29-41. [CrossRef]

32. Yamasaki, K.; Kigawa, T.; Inoue, M.; Tateno, M.; Yamasaki, T.; Yabuki, T.; Aoki, M.; Seki, E.; Matsuda, T.; Nunokawa, E.; et al. A novel zinc-binding motif revealed by solution structures of DNA-binding domains of Arabidopsis SBP-family transcription factors. J. Mol. Biol. 2004, 337, 49-63. [CrossRef] [PubMed]

33. Wu, G.; Park, M.Y.; Conway, S.R.; Wang, J.-W.; Weigel, D.; Poethig, R.S. The Sequential Action of miR156 and miR172 Regulates Developmental Timing in Arabidopsis. Cell 2009, 138, 750-759. [CrossRef] [PubMed]

34. Schwarz, S.; Grande, A.V.; Bujdoso, N.; Saedler, H.; Huijser, P. The microRNA regulated SBP-box genes SPL9 and SPL15 control shoot maturation in Arabidopsis. Plant Mol. Biol. 2008, 67, 183-195. [CrossRef] 
35. Jung, J.-H.; Lee, H.-J.; Ryu, J.Y.; Park, C.-M. SPL3/4/5 Integrate Developmental Aging and Photoperiodic Signals into the FT-FD Module in Arabidopsis Flowering. Mol. Plant 2016, 9, 1647-1659. [CrossRef]

36. Hou, H.; Yan, X.; Sha, T.; Yan, Q.; Wang, X. The SBP-Box Gene VpSBP11 from Chinese Wild Vitis Is Involved in Floral Transition and Affects Leaf Development. Int. J. Mol. Sci. 2017, 18, 1493. [CrossRef]

37. Yu, N.; Niu, Q.-W.; Ng, K.-H.; Chua, N.-H. The role of miR156/SPLs modules in Arabidopsis lateral root development. Plant J. 2015, 83, 673-685. [CrossRef]

38. Xing, S.; Salinas, M.; Hoehmann, S.; Berndtgen, R.; Huijser, P. miR156-Targeted and Nontargeted SBP-Box Transcription Factors Act in Concert to Secure Male Fertility in Arabidopsis. Plant Cell 2010, 22, 3935-3950. [CrossRef]

39. Ferreira e Silva, G.F.; Silva, E.M.; Azevedo, M.d.S.; Corazon Guivin, M.A.; Ramiro, D.A.; Figueiredo, C.R.; Carrer, H.; Pereira Peres, L.E.; Silveira Nogueira, F.T. microRNA156-targeted SPL/ SBP box transcription factors regulate tomato ovary and fruit development. Plant J. 2014, 78, 604-618. [CrossRef] [PubMed]

40. Liu, Y.; Zhao, Q.; Meng, N.; Song, H.; Li, C.; Hu, G.; Wu, J.; Lin, S.; Zhang, Z. Over-expression of EjLFY-1 Leads to an Early Flowering Habit in Strawberry (Fragaria x ananassa) and Its Asexual Progeny. Front. Plant Sci. 2017, 8. [CrossRef]

41. Shikata, M.; Koyama, T.; Mitsuda, N.; Ohme-Takagi, M. Arabidopsis SBP-Box Genes SPL10, SPL11 and SPL2 Control Morphological Change in Association with Shoot Maturation in the Reproductive Phase. Plant Cell Physiol. 2009, 50, 2133-2145. [CrossRef] [PubMed]

42. Yu, S.; Galvao, V.C.; Zhang, Y.-C.; Horrer, D.; Zhang, T.-Q.; Hao, Y.-H.; Feng, Y.-Q.; Wang, S.; Schmid, M.; Wang, J.-W. Gibberellin Regulates the Arabidopsis Floral Transition through miR156-Targeted SQUAMOSA PROMOTER BINDING-LIKE Transcription Factors. Plant Cell 2012, 24, 3320-3332. [CrossRef] [PubMed]

43. Chuck, G.; Whipple, C.; Jackson, D.; Hake, S. The maize SBP-box transcription factor encoded by tasselsheath4 regulates bract development and the establishment of meristem boundaries. Development 2010, 137, 1243-1250. [CrossRef] [PubMed]

44. Hou, H.; Jia, H.; Yan, Q.; Wang, X. Overexpression of a SBP-Box Gene (VpSBP16) from Chinese Wild Vitis Species in Arabidopsis Improves Salinity and Drought Stress Tolerance. Int. J. Mol. Sci. 2018, 19, 940. [CrossRef] [PubMed]

45. Lin, S.; Sharpe, R.H.; Janick, J. Loquat: Botany and Horticulture. In Horticultural Reviews; Janick, J., Ed.; Wiley: Hoboken, NJ, USA, 1999; Volume 23. [CrossRef]

46. Esumi, T.; Tao, R.; Yonemori, K. Isolation of LEAFY and TERMINAL FLOWER 1 homologues from six fruit tree species in the subfamily Maloideae of the Rosaceae. Sex. Plant Reprod. 2005, 17, 277-287. [CrossRef]

47. Liu, Y.; Song, H.; Liu, Z.; Hu, G.; Lin, S. Molecular characterization of loquat EjAP1 gene in relation to flowering. Plant Growth Regul. 2013, 70, 287-296. [CrossRef]

48. Zhang, L.; Yu, H.; Lin, S.; Gao, Y. Molecular Characterization of FT and FD Homologs from Eriobotrya deflexa Nakai forma koshunensis. Front. Plant Sci. 2016, 7, 8. [CrossRef]

49. Zhang, L.; Jiang, Y.; Zhu, Y.; Su, W.; Long, T.; Huang, T.; Peng, J.; Yu, H.; Lin, S.; Gao, Y. Functional characterization of GI and CO homologs from Eriobotrya deflexa Nakai forma koshunensis. Plant Cell Rep. 2019, 38, 533-543. [CrossRef]

50. Jiang, Y.; Peng, J.; Zhu, Y.; Su, W.; Zhang, L.; Jing, Y.; Lin, S.; Gao, Y. The Role of EjSOC1s in Flower Initiation in Eriobotrya japonica. Front. Plant Sci. 2019, 10, 253. [CrossRef]

51. Yamaguchi, A.; Wu, M.-F.; Yang, L.; Wu, G.; Poethig, R.S.; Wagner, D. The MicroRNA-Regulated SBP-Box Transcription Factor SPL3 Is a Direct Upstream Activator of LEAFY, FRUITFULL, and APETALA1. Dev. Cell 2009, 17, 268-278. [CrossRef]

52. Huijser, P.; Schmid, M. The control of developmental phase transitions in plants. Development 2011, 138, 4117-4129. [CrossRef] [PubMed]

53. Fan, S.; Zhang, D.; Gao, C.; Wan, S.; Lei, C.; Wang, J.; Zuo, X.; Dong, F.; Li, Y.; Shah, K.; et al. Mediation of Flower Induction by Gibberellin and its Inhibitor Paclobutrazol: mRNA and miRNA Integration Comprises Complex Regulatory Cross-Talk in Apple. Plant Cell Physiol. 2018, 59, 2288-2307. [CrossRef] [PubMed]

54. Shalom, L.; Shlizerman, L.; Zur, N.; Doron-Faigenboim, A.; Blumwald, E.; Sadka, A. Molecular characterization of SQUAMOSA PROMOTER BINDING PROTEIN-LIKE (SPL) gene family from Citrus and the effect of fruit load on their expression. Front. Plant Sci. 2015, 6, 389. [CrossRef] [PubMed] 
55. Song, C.N.; Qian, J.L.; Fang, J.G.; Wang, H.K.; Qiu, X.L.; Zhang, Z.; Zhang, X.Y. Cloning, Subcellular Localization and Expression Analysis of SPL9 and SPL13 Genes from Poncirus trifoliata. Sci. Agric. Sin. 2010, 43, 2105-2114.

56. Gandikota, M.; Birkenbihl, R.P.; Hoehmann, S.; Cardon, G.H.; Saedler, H.; Huijser, P. The miRNA156/157 recognition element in the 3' UTR of the Arabidopsis SBP box gene SPL3 prevents early flowering by translational inhibition in seedlings. Plant J. 2007, 49, 683-693. [CrossRef] [PubMed]

57. Jung, J.-H.; Seo, P.J.; Kang, S.K.; Park, C.-M. miR172 signals are incorporated into the miR156 signaling pathway at the SPL3/4/5 genes in Arabidopsis developmental transitions. Plant Mol. Biol. 2011, 76, 35-45. [CrossRef]

58. Wei, Q.; Ma, C.; Xu, Y.; Wang, T.; Chen, Y.; Lu, J.; Zhang, L.; Jiang, C.-Z.; Hong, B.; Gao, J. Control of chrysanthemum flowering through integration with an aging pathway. Nat. Commun. 2017, 8, 829. [CrossRef]

59. Long, J.-M.; Liu, C.-Y.; Feng, M.-Q.; Liu, Y.; Wu, X.-M.; Guo, W.-W. miR156-SPL modules regulate induction of somatic embryogenesis in citrus callus. J. Exp. Bot. 2018, 69, 2979-2993. [CrossRef]

60. Silva, G.F.F.; Silva, E.M.; Correa, J.P.O.; Vicente, M.H.; Jiang, N.; Notini, M.M.; Junior, A.C.; De Jesus, F.A.; Castilho, P.; Carrera, E.; et al. Tomato floral induction and flower development are orchestrated by the interplay between gibberellin and two unrelated microRNA-controlled modules. New Phytol. 2019, 221, 1328-1344. [CrossRef]

61. Negishi, K.; Endo, M.; Abe, M.; Araki, T. SODIUM POTASSIUM ROOT DEFECTIVE1 regulates FLOWERING LOCUS T expression via the microRNA156-SQUAMOSA PROMOTER BINDING PROTEIN-LIKE3 module in response to potassium conditions. Plant Cell Physiol. 2018, 59, 404-413. [CrossRef]

62. Reig, C.; Gil-Munoz, F.; Vera-Sirera, F.; Garcia-Lorca, A.; Martinez-Fuentes, A.; Mesejo, C.; Perez-Amador, M.A.; Agusti, M. Bud sprouting and floral induction and expression of FT in loquat [Eriobotrya japonica (Thunb.) Lindl.]. Planta 2017, 246, 915-925. [CrossRef] [PubMed]

63. Shan, L.L.; Li, X.; Wang, P.; Cai, C.; Zhang, B.; De Sun, C.; Zhang, W.S.; Xu, C.J.; Ferguson, I.; Chen, K.S. Characterization of cDNAs associated with lignification and their expression profiles in loquat fruit with different lignin accumulation. Planta 2008, 227, 1243-1254. [CrossRef] [PubMed]

64. Livak, K.J.; Schmittgen, T.D. Analysis of relative gene expression data using real-time quantitative PCR and the 2-DELTADELTACT method. Methods 2001, 25, 402-408. [CrossRef] [PubMed]

65. Lee, L.Y.C.; Hou, X.; Fang, L.; Fan, S.; Kumar, P.P.; Yu, H. STUNTED mediates the control of cell proliferation by GA in Arabidopsis. Development 2012, 139, 1568-1576. [CrossRef]

66. Sparkes, I.A.; Runions, J.; Kearns, A.; Hawes, C. Rapid, transient expression of fluorescent fusion proteins in tobacco plants and generation of stably transformed plants. Nat. Protoc. 2006, 1, 2019-2025. [CrossRef]

67. Hou, X.; Zhou, J.; Liu, C.; Liu, L.; Shen, L.; Yu, H. Nuclear factor Y-mediated H3K27me3 demethylation of the SOC1 locus orchestrates flowering responses of Arabidopsis. Nat. Commun. 2014, 5, 4601. [CrossRef]

68. Zhang, X.; Henriques, R.; Lin, S.-S.; Niu, Q.-W.; Chua, N.-H. Agrobacterium-mediated transformation of Arabidopsis thaliana using the floral dip method. Nat. Protoc. 2006, 1, 641-646. [CrossRef]

69. Hellens, R.P.; Allan, A.C.; Friel, E.N.; Bolitho, K.; Grafton, K.; Templeton, M.D.; Karunairetnam, S.; Gleave, A.P.; Laing, W.A. Transient expression vectors for functional genomics, quantification of promoter activity and RNA silencing in plants. Plant Methods 2005, 1, 13. [CrossRef]

70. Moyle, R.L.; Carvalhais, L.C.; Pretorius, L.-S.; Nowak, E.; Subramaniam, G.; Dalton-Morgan, J.; Schenk, P.M. An Optimized Transient Dual Luciferase Assay for Quantifying MicroRNA Directed Repression of Targeted Sequences. Front. Plant Sci. 2017, 8, 1631. [CrossRef]

(C) 2019 by the authors. Licensee MDPI, Basel, Switzerland. This article is an open access article distributed under the terms and conditions of the Creative Commons Attribution (CC BY) license (http://creativecommons.org/licenses/by/4.0/). 